\title{
Wind speed prediction research with EMD-BP based on Lorenz disturbance
}

\author{
Yagang Zhang ${ }^{*, * *}$, Guifang Pan ${ }^{*}$, Chenhong Zhang ${ }^{*}$ Yuan Zhao*
}

\begin{abstract}
Wind power, as a new energy generation technology, has been applying widely and growing rapidly, which make it become the main force of renewable energy. However, wind speed sequence has its own character of the intermittent and uncertainty, which brings a great challenge to the safety and stability of the power grid, one of the valid ways solving the problem is improving the wind speed predicting accuracy. Therefore, given atmospheric disturbances, we firstly used empirical mode decomposition (EMD) to deal with the non-linear wind speed sequence, and combined with strong adaptive and self-learning ability of BP neural network, then, a wind speed prediction model, EMD-BP neural network based on Lorenz disturbance, was proposed. Finally, it was to made use of actual wind speed data to take a simulation experiment and explored the improvement effect of the preliminary forecasting sequence of wind speed influenced by Lorenz equation in the transient chaos and chaos. The results show that, the improved model weakened the random fluctuation of wind speed sequence, effectively corrected the wind speed sequences initial prediction values, and made a great improvement for the short-term wind speed prediction precision. This research work will help the power system dispatching department adjust the dispatching plan in time, formulate the wind farm control strategy reasonably, reduce the impact brought by wind power grid connection, increase the wind power penetration rate, and then promote the global energy power market innovation.
\end{abstract}

K e y w ords: short-term wind speed prediction, renewable energy, Lorenz system, empirical mode decomposition, BP neural network

\section{Introduction}

Excessive consumption of fossil fuels has led to a sharp drop in fossil energy reserves, aggravating environmental pollution and abnormal weather. Developing and utilizing clean renewable energy, and promoting countries towards low-carbon and sustainable energy systems, are effective ways to cope with the energy crisis and environmental crisis.

At present, wind power as a green renewable energy generation technology has been applied in the world. According to the global wind energy council (GWEC) in 2018 [1], in 2017, the wind power newly installed capacity was $52.57 \mathrm{GW}$, and the cumulative installed capacity was $539.581 \mathrm{GW}$, of which Chinas newly installed capacity reached $19.5 \mathrm{GW}$, accounting for $37.1 \%$ of the total newly installed capacity, leading the world. In recent years, the offshore wind power installed capacity is on a steady increase, it has increased by $4.331 \mathrm{GW}$ in 2017. The cumulative installed capacity has reached $18.814 \mathrm{GW}$, a $30 \%$ increase over the cumulative installed capacity in 2016. However, wind power safety gridconnected still faces some challenges, which is including the instability of its output power and its influence on power system operation. Accurate wind power prediction is beneficial to the power dispatching department to make the overall scheduling plan, alleviate the influence of wind power instability on the power grid, and guarantee the balance of supply and demand in the power grid. In the meantime, wind power forecasting accuracy is also the key to reduce the cost of wind power generation and keep competitiveness in the power market [2]. Therefore, the research of wind power development mostly focuses on improving prediction methods of wind speed and power, among which, wind speed prediction is not only basic work but also key link.

The characteristics of the prediction object itself, data processing methods and the prediction model performance determine the wind speed prediction accuracy. The random fluctuation characteristics that the wind speed series show have a great influence on the prediction accuracy. The larger the fluctuation amplitude is, the low the prediction accuracy will be. Preprocessing the wind speed series can mine the hidden information of the series and reduce the complexity of the series, and improve the prediction model performance. Common data processing methods include wavelet decomposition $[3,4]$, principal component analysis [5], independent component analysis [6], and empirical mode decomposition [7]. At this stage, commonly used wind speed prediction methods include artificial neural networks [8-10], grey prediction $[11,12]$, time series analysis [13-14], support vector

*State Key Laboratory of Alternate Electrical Power System with Renewable Energy Sources, North China Electric Power University, Beijing, 102206, China, ** Interdisciplinary Mathematics Institute, University of South Carolina, Columbia, SC 29208, United States, yagangzhang@ncepu.edu.cn

DOI: $10.2478 /$ jee-2019-0028, Print (till 2015) ISSN 1335-3632, On-line ISSN 1339-309X

(C) This is an open access article licensed under the Creative Commons Attribution-NonCommercial-NoDerivs License (http://creativecommons.org/licenses/by-nc-nd/3.0/). 
machines $[15,16]$, and so on, in which, artificial neural network not only has nonlinear fitting ability, but also has the adaptive and self-learning characteristic, especially suitable for wind power prediction.

The wind formation is a typical nonlinear process in the atmospheric system, and its inherent volatility brings many difficulties to wind speed prediction. The atmospheric dynamical system is a certain system, whose motion change laws are explained by a set of Lorenz equation. Changing the Rayleigh number of this equation, the Lorenz attractor will exhibit four different motion patterns. Reference [17] verifies the effectiveness and feasibility of Lorenzs atmospheric disturbance system in improving wind speed prediction accuracy. Reference [18] discusses the compensation effect on the preliminary wind speed forecast results using atmospheric disturbances with different initial values. The Lorenz atmospheric disturbance system has unique advantages in increasing the wind speed forecasting accuracy. Thus, in the prediction work, based on the Lorenz atmospheric disturbance system, we consider changing the Rayleigh number, and study the compensation effect the compensation effect of the Lorenz atmospheric disturbance system under transient chaos and chaos on the initial wind speed prediction value.

Simultaneously, Taking account of the strong nonlinear characteristics of the wind speed sequence, we established a wind speed prediction model with EMD-BP based on Lorenz system. That is, through empirical mode decomposition, the wind speed sequence can firstly be decomposed into several empirical modal functions reflecting the signal different scale characteristics and a residual term that represents a signal change trend, then, it is to make a linear prediction for the remaining terms and build the BP neural network prediction model for each empirical mode function, which will greatly improve the BP neural network prediction performance, finally, Lorenz equation is used as an atmospheric disturbance model to investigate the improving effect in different atmospheric motion states on wind speed prediction precise.

\section{Empirical mode decomposition and Lorenz system}

\subsection{Empirical mode decomposition (EMD)}

Based on the local characteristics of the signal sequence time scale, EMD can adaptively decomposes the signal without any basis functions pre-set. This is essentially different from the Fourier decomposition and wavelet decomposition methods. EMD is has unique advantages in the processing of nonlinear and nonstationary signals [19].

For a given original wind speed series $\{x(t)\}$, the steps for empirical mode decomposition are as follows:

(1) Get the position and amplitude of all local maximum and minimum values in the signal;
(2) The local maxima spline interpolation consists of the upper envelope, and the local minima spline interpolation composes the lower envelope, denoted by $e_{\max }$ and $e_{\min }$

(3) In each period $t$, calculating the average value of upper and lower enveloping curve

$$
u_{1}=\frac{e_{\max }+e_{\min }}{2} ;
$$

(4) Subtracting the input signal in the average enveloping line, namely $l_{1}(t)=x(t)-u_{1}(t)$, which is the iteration of the filtering process. The next step is checking if $l_{1}(t)$ is an intrinsic modal function. While the difference of two consecutive filters is less than a selected threshold SD, the filtering process is stopped, and SD is defined as

$$
S D=\sum_{t=0}^{T}\left[\frac{\left|l_{1(k-1)}(t)-l_{1 k}(t)\right|^{2}}{l_{1(k-1)}^{2}(t)}\right]
$$

where $l_{11}(t)=l_{1}(t)-u_{11}(t)$ and if this is $\mathrm{k}$ iterations, then $l_{1 n}(t)=l_{1(n-1)}(t)-l_{1 n}(t)$.

(5) When the condition is satisfied, the intrinsic modal function is defined as $g_{1}(t)=l_{1 n}(t)$.

After obtaining the intrinsic modal function $g_{1}(t)$, we define the remainder as

$$
s_{1}(t)=x(t)-g_{1}(t) .
$$

(6) Take the above residual as the input signal, then repeat the steps $(1)-(6)$. Therefore, the result is

$$
s_{2}(t)=s_{1}(t)-g_{2}(t), \ldots, s_{n}(t)=s_{n-1}(t)-g_{n}(t) .
$$

When the residual does not contain any extreme points, the empirical mode decomposition process is completed. This means that the residual value can be a constant or a monotonic function. The signal could be expressed as the sum of the intrinsic modal function and the final residual value

$$
x(t)=\sum_{i=1}^{n} g_{i}(t)+s_{n}(t) .
$$

\subsection{Lorenz system}

In the 1960s, when the American meteorologist E. N. Lorenz studied the regional microclimate, starting from the fluid convection model he extracted an atmospheric convention model to represent the same motion state, which is the famous Lorenz dynamic system. It is regarded as the first dynamic system representing chaotic state in the simplest way [18]. At the same time, the differential equation (5) describes the chaotic state of the nonlinear dynamical system in the easiest way [22].

$$
\left\{\begin{array}{l}
\dot{x}=-\sigma(x-y) \\
\dot{y}=-x y+r x-y, \\
\dot{z}=x y-b z
\end{array}\right.
$$




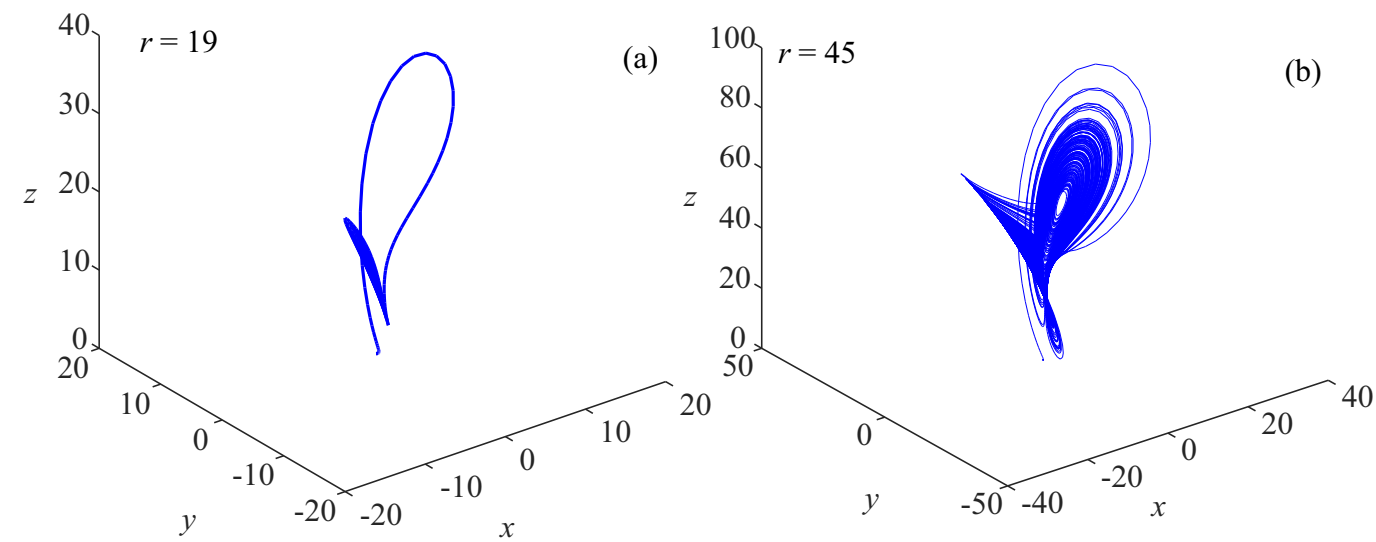

Fig. 1. Three-dimensional phase diagram in transient chaotic and chaotic(Lorenz attractor): (a) - the Lorentz attractor motion pattern when $r=19$, (b) - the Lorentz attractor motion pattern when $r=45$

Table 1. The actual fluid motion of the Lorenz system in the different Rayleigh numbers

\begin{tabular}{ccccc}
\hline$r$ & $0<r<1$ & $r<13.9656$ & $13.9656<r<24.06$ & $r>24.06$ \\
Actual & Heat & Convection & Regular & Irregular \\
fluid motion & conduction & motion & convection $*$ & turbulent motion \\
\hline
\end{tabular}

* after a long period of transient chaos

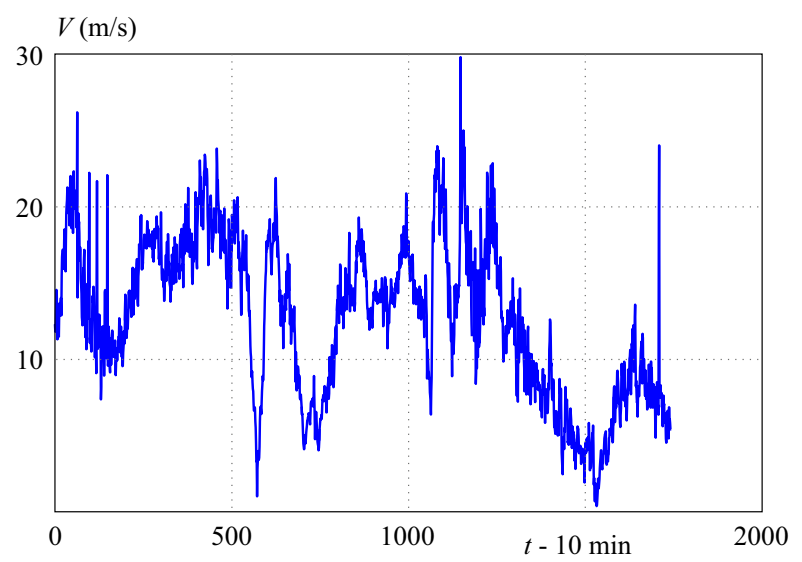

Fig. 2. The actual wind speed distribution recorded every $10 \mathrm{~min}-$ utes in Chinese wind farm

In the formula, the state variable $x, y, z$, respectively represent the convection motion amplitude, the temperature difference between the rising and falling fluids in the horizontal direction, and the deviation from the linear case by the vertical temperature difference due to convection. Parameters $\sigma, r, b$ are all the dimensionless positive value, indicating the Prandtl number, Rayleigh number and the parameter related to the size of regional climate respectively.

Referencing the research of meteorologists Saltzman and Lorenz, fixing the parameters $\sigma, b$ as 10 and 8/3, and making the initial value of the Lorenz equation unchanged, it is changing the Prandtl number $r$ to observe how the Lorenz attractor motion state changes. The actual fluid motion of the Lorenz system in the different Rayleigh numbers is shown in Tab. 1 [23].

The wind formation is a very typical nonlinear process, so in this paper, we focus on the corrected effect of the Lorenz disturbance sequence in the transient chaos and chaos on the wind speed forecasting result. Figure 1 is the motion of Lorenz attractor in the case that the initial condition is $(0.01,1,1)$, the parameters are $\sigma=10$, $b=\frac{8}{3}$, and the Rayleigh number is respectively $r=19$, $r=45$. As it is shown in Fig.1, in the same initial condition, Lorenz attractor in the transient chaos and chaos exhibit completely different motions.

\section{The modeling process of EMD-BP wind speed forecasting model based on Lorenz disturbance}

Wind speed sequence is a set of complex nonlinear time series, which presents not only strong non-stationary, but periodic variation laws with nesting different cycles. Empirical mode decomposition (EMD) method with good time-frequency localization properties, can adaptively decompose the original wind speed sequence into intrinsic modal functions with local time-varying characteristics and a residual, and better reveal the internal changing rules of signals. The BP neural network with a strong nonlinear fitting ability, can map the complex nonlinear relationship at random, which is a significant wind speed prediction modeling method. Lorenz System restores atmospheric motion status and effectively compensates pre- 


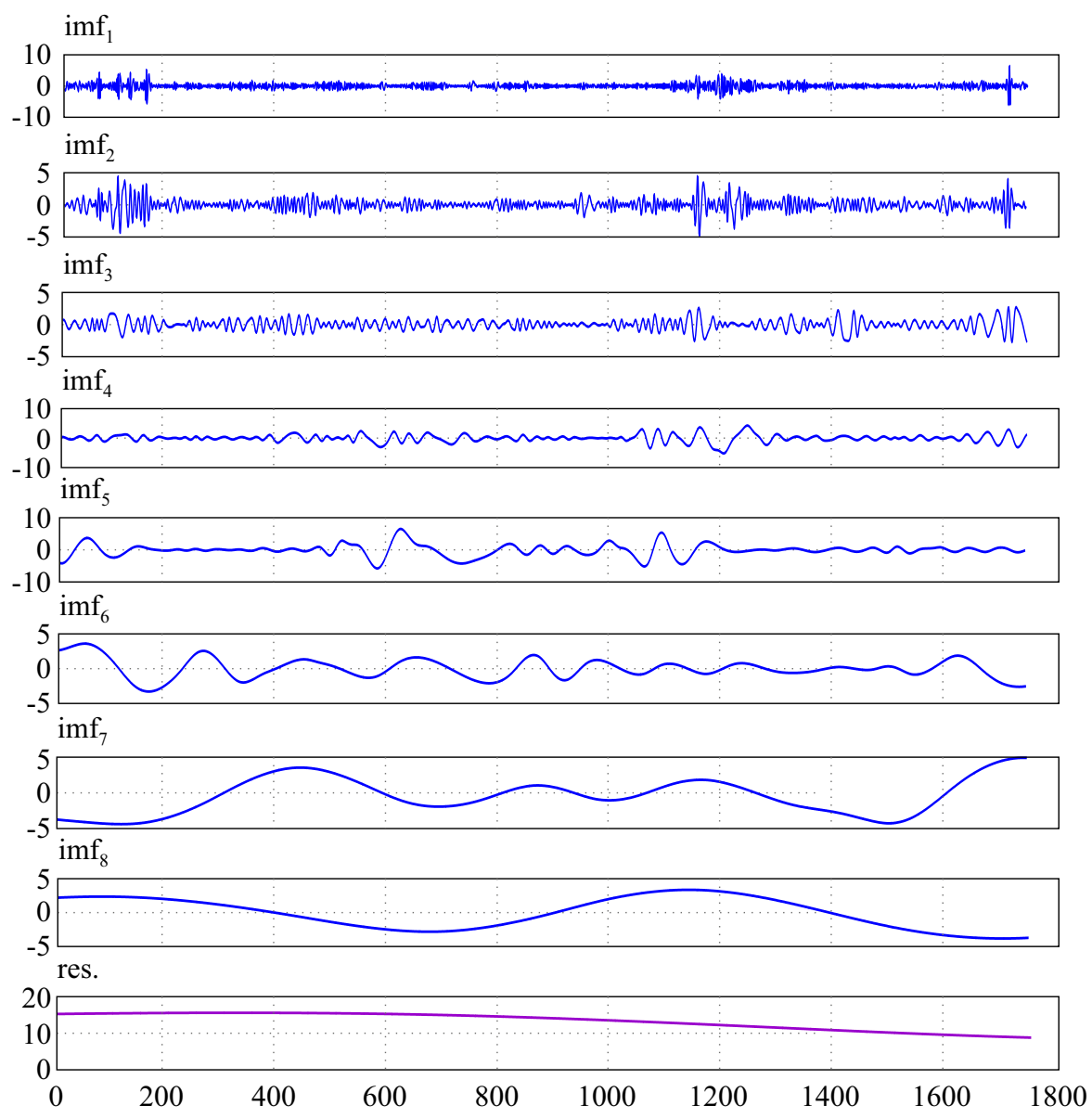

Fig. 3. The EMD decomposition results

liminary wind speed prediction results. In summary, in this section, we combine the advantages of empirical mode decomposition, BP neural network and Lorenz system to establish the wind speed prediction model.

\subsection{Data description}

Wind speed data of Chinese wind farm is adopted to carry out simulation experiment and verify the adaptability of the research method for real wind speed sequences. Figure 2 is wind speed distribution during a certain period in China. From the figure, we see that, the maximum wind speed is close to $30 \mathrm{~m} / \mathrm{s}$, but the minimum is only close to $0 \mathrm{~m} / \mathrm{s}$, and the wind speed fluctuates very much, obviously showing strong random fluctuations.

\subsection{The EMD decomposition results}

The original wind speed data was processed by empirical mode decomposition, and the decomposition result is shown in Fig. 3. The initial sequence was broken into nine components. From IMF1 to IMF8 is the intrinsic mode function, whose frequency fluctuation reduced in turn, and the fluctuation range is smaller than the original sequence, in addition, it reflects different scale features of wind speed sequences. The purple curve in the last graph of Fig. 3 indicates the residual term, describing the wind speed trend information.

\subsection{The wind speed prediction model of EMD-BP neu- ral network}

As Fig. 3 shows, eight intrinsic mode functions has nonlinear characteristics. So, the BP neural network with strong nonlinear fitting ability is used to establish the appropriate three-layer neural network for each intrinsic modal function, getting the forecast result of each IMF component. Fitting the true and measured values of each component to test the BP neural network prediction ability. The real value and measured value of each component are fitted to test the predicting capacity of BP neural network, and the fitting effect is shown in Fig. 4. The horizontal axis indicates the true value. As Fig 4 shows, the vertical axis is the predicted value, the green plus sign is the predicted value, and the black straight line is the regression line. Graphs (a)-(h) from Fig. 4 describe the fitting effect between forecasting values and real values of the IMF component, the effect is better with the sequence fluctuation becoming smaller, That is to say, the prediction ability of BP neural network becomes better with the sequence fluctuation becoming smaller. The fitting effect of between forecasting values and real values of the wind 


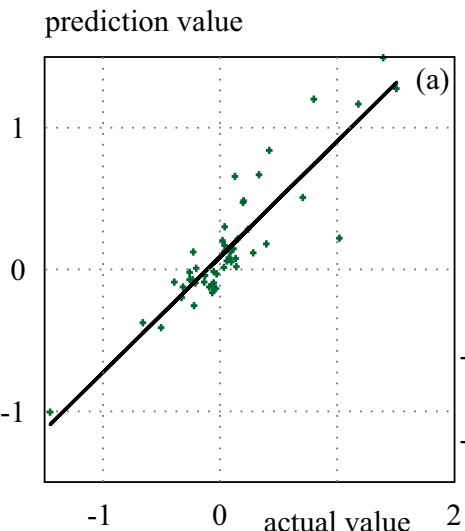

prediction value

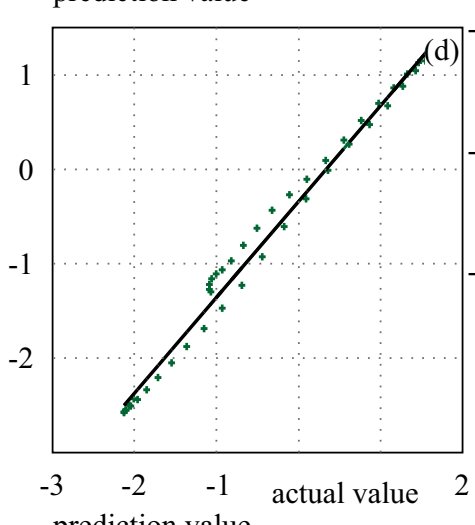
prediction value

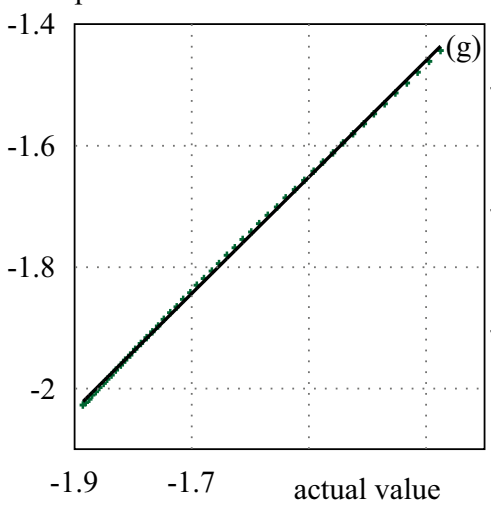

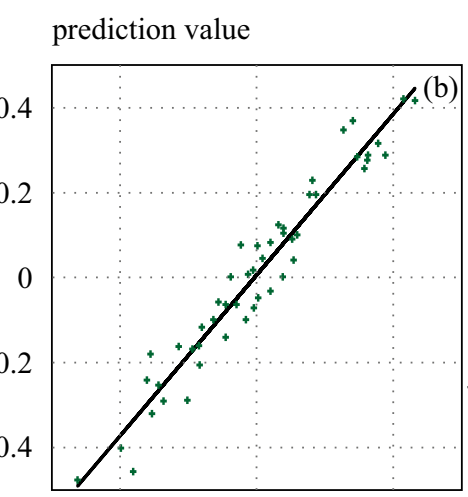

$-0.4 \quad 0$ actual value prediction value

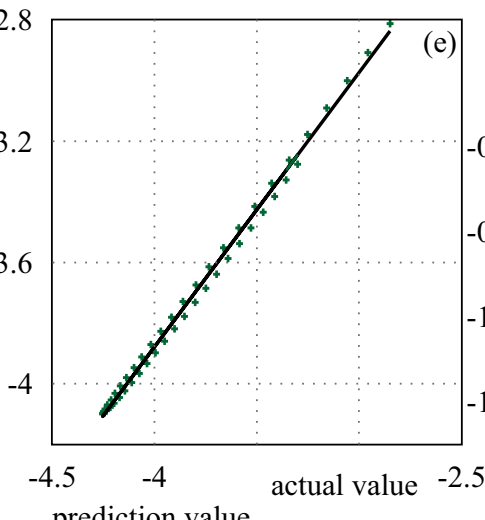

prediction value

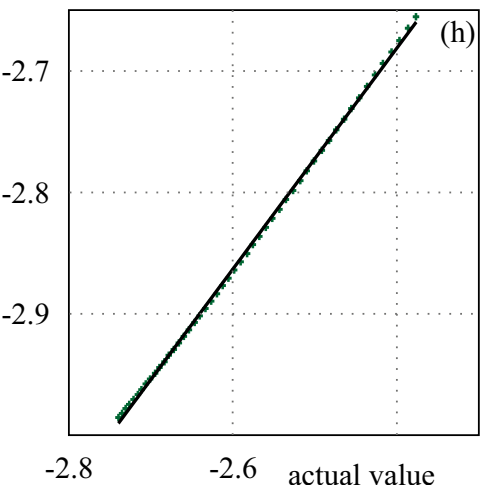

prediction value

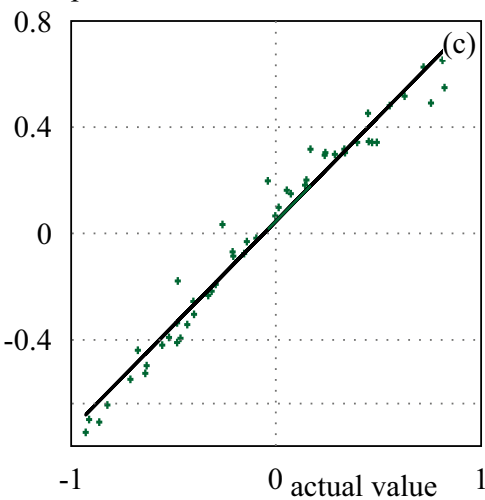

prediction value

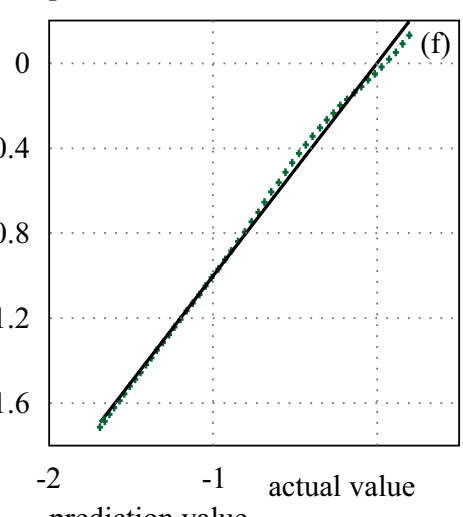

prediction value

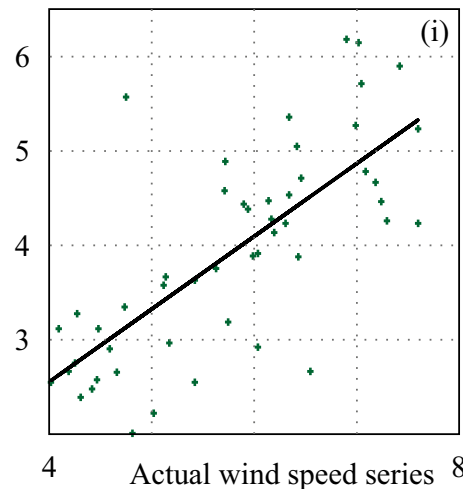

Fig. 4. The fitting curve of real values and predicted values; (a)-(h) are fitting images of decomposed values and predicted values; (i) are the fitting images of measured values and predicted values
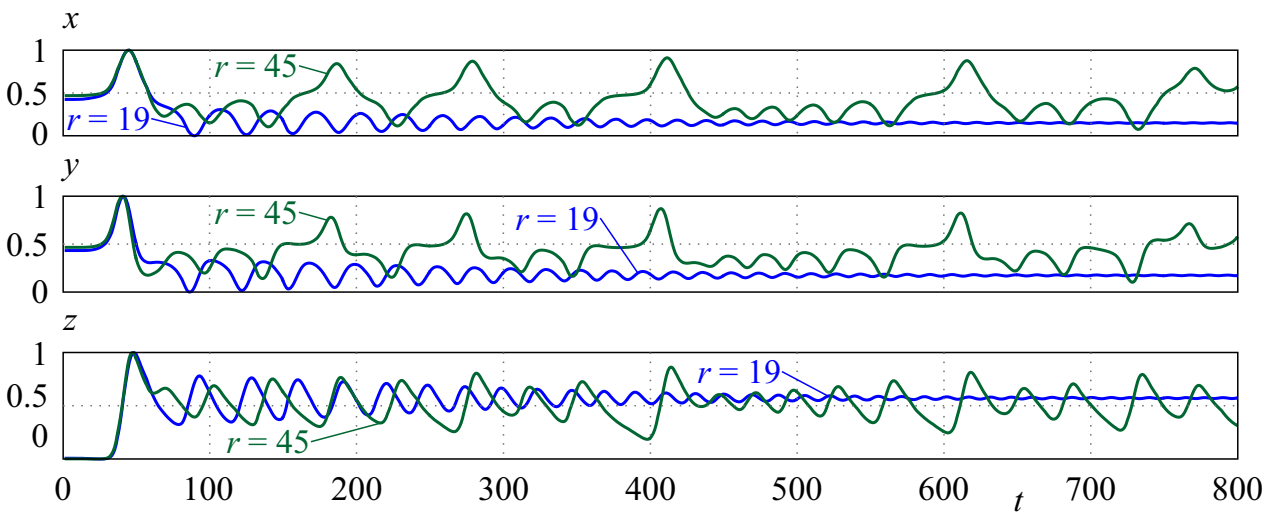

Fig. 5. The time series curve of variables $x, y, z$, each time series contains 800 time units 


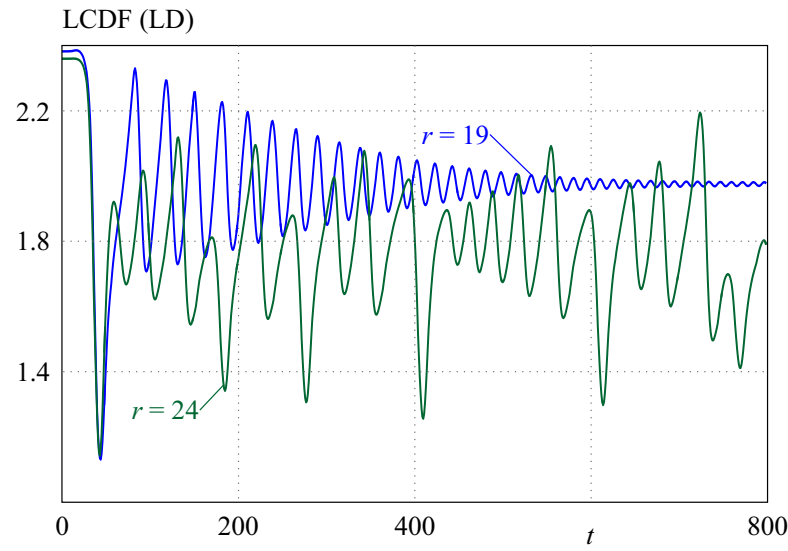

Fig. 6. The Lorenz comprehensive disturbance series produced by the Lorenz equation and the Lance-Williams distance

speed sequence is shown in graph (i), the dispersed range of the prediction result is pretty large, and is not close to the regression curve, so the BP neural network's prediction ability for each component is significantly higher than that of the original wind speed sequence.

\subsection{The Lorenz comprehensive disturbance flow}

From Fig. 1, it can be seen clearly that Lorenz attractor in transient chaos and chaos have different forms under the same initial conditions. The wind formation shows typical nonlinear feature. Therefore, in the different motion state of transient chaos and chaos, we investigate the compensation effect of Lorenz disturbance sequence on the initial wind speed prediction values.

\subsubsection{Solving the Lorenz equation}

Fixed the initial value is $C_{0}(0.01,1,1)$, parameter $\sigma=10, b=\frac{8}{3}$, let Rayleigh number $r=19$ and $r=45$ to determine the two kinds of motion patterns: the transient chaos and the chaos, and obtain two sets of disturbance variables, as shown in Fig. 5. It can be seen that under the transient chaotic and chaotic states, each component exhibits random fluctuation characteristics and changes with time and the curves are very different.

\subsubsection{The formation of the Lorenz comprehensive disturbance flow (L C D F)}

The wind speed is a positive number, and we solve the Lorenz equation to get a three-dimensional random variable, that is the Lorenz disturbance variable. In order to solve the problem of non-uniform dimensions, we introduce Lance distance (LD) as a mapping function to reduce the dimension of disturbance variables. It can map the three-dimensional disturbance variable to the distance function, which calls the Lorenz comprehensive disturbance flow (LCDF). To eliminate the influence of three disturbance variable in different dimension, data need to be standardized before calculating Lorenz comprehensive disturbance flow $[24,25]$. The standardized formula is as follows

$$
\breve{x}_{t}=\frac{x_{t}-\bar{x}}{\sigma_{x}}, \quad \breve{y}_{t}=\frac{y_{t}-\bar{y}}{\sigma_{y}}, \quad \breve{z}=\frac{z_{t}-\bar{z}}{\sigma_{z}}
$$

where the numerical solution of the Lorenz equation is expressed as $x_{t}, y_{t}, z_{t}, t=1,2, \ldots, n, \bar{x}, \bar{y}, \bar{z}$ indicate the mean value, and $\sigma_{x}, \sigma_{y}, \sigma_{z}$ indicate the standard deviation. In order to facilitate the unification of the written symbols, the standardized data $\left(\breve{x}_{t}, \breve{y}_{t}, \breve{z}_{t}\right)$ is marked as $\left(x_{t}, y_{t}, z_{t}\right)$. The Lance-Williams distance is defined as

$$
d_{L}\left(C_{t}, C_{0}\right)=\frac{\left|x_{t}-x_{0}\right|}{x_{t}+x_{0}}+\frac{\left|y_{t}-y_{0}\right|}{y_{t}+y_{0}}+\frac{\left|z_{t}-z_{0}\right|}{z_{t}+z_{0}}
$$

where the motion state at a certain moment in the Lorenz system is $C_{t}\left(x_{t}, y_{t}, z_{t}\right)$, and $C_{0}\left(x_{0}, y_{0}, z_{0}\right)$ is the initial value.

Based on the formulae (5)-(7), the three-dimensional disturbance variables in the Lorenz system will be integrated into one-dimensional random variables, resulting in Lorenz Comprehensive Disturbance Flow (LCDF), the distribution is shown in Fig. 6. The LCDF sequence exhibits random fluctuation characteristics.

\subsection{The modeling steps EMD-BP of emd-bp wind speed forecasting model based on Lorenz disturbance}

Based on the above EMD-BP wind speed forecasting model and Lorenz atmospheric disturbance system, we will obtain a detailed modeling process for the EMD$\mathrm{BP}$ wind speed forecasting model based on the Lorenz disturbance, and named this model as the LD-EMD-BP model.

Step 1: The original wind speed sequence decomposition. Using EMD method decompose the original wind speed series $\{x(t)\}$ to obtain several IMF components with different frequencies and the remainder $r(t)$ reflecting the trend of wind speed series;

Step 2: The IMF Components prediction. According to each IMF component characteristic, the optimal BP parameters are respectively searched to establish the $\mathrm{BP}$ model that can best fit the IMF component, and each IMF component is predicted to obtain the forecasting value $y_{1}, y_{2}, \ldots, y_{m}$

Step 3: The remainder $r(t)$ prediction. The remainder $r(t)$ belongs to a more stable time series and is nearly linear. Therefore, the linear prediction model is used to fit the remainder to obtain the predicted value $y_{m+1}$;

Step 4: The initial wind speed prediction value is obtained by superimposing the IMF component prediction value and the remainder prediction value $v_{t}$;

Step 5: The correction influence of the Lorenz integrated perturbation flow on $v_{t}$. In order to minimize the root mean square error (RMSE) between the predicted series and the real series, a disturbance modification formula is used to impose a disturbance enhancement on the 


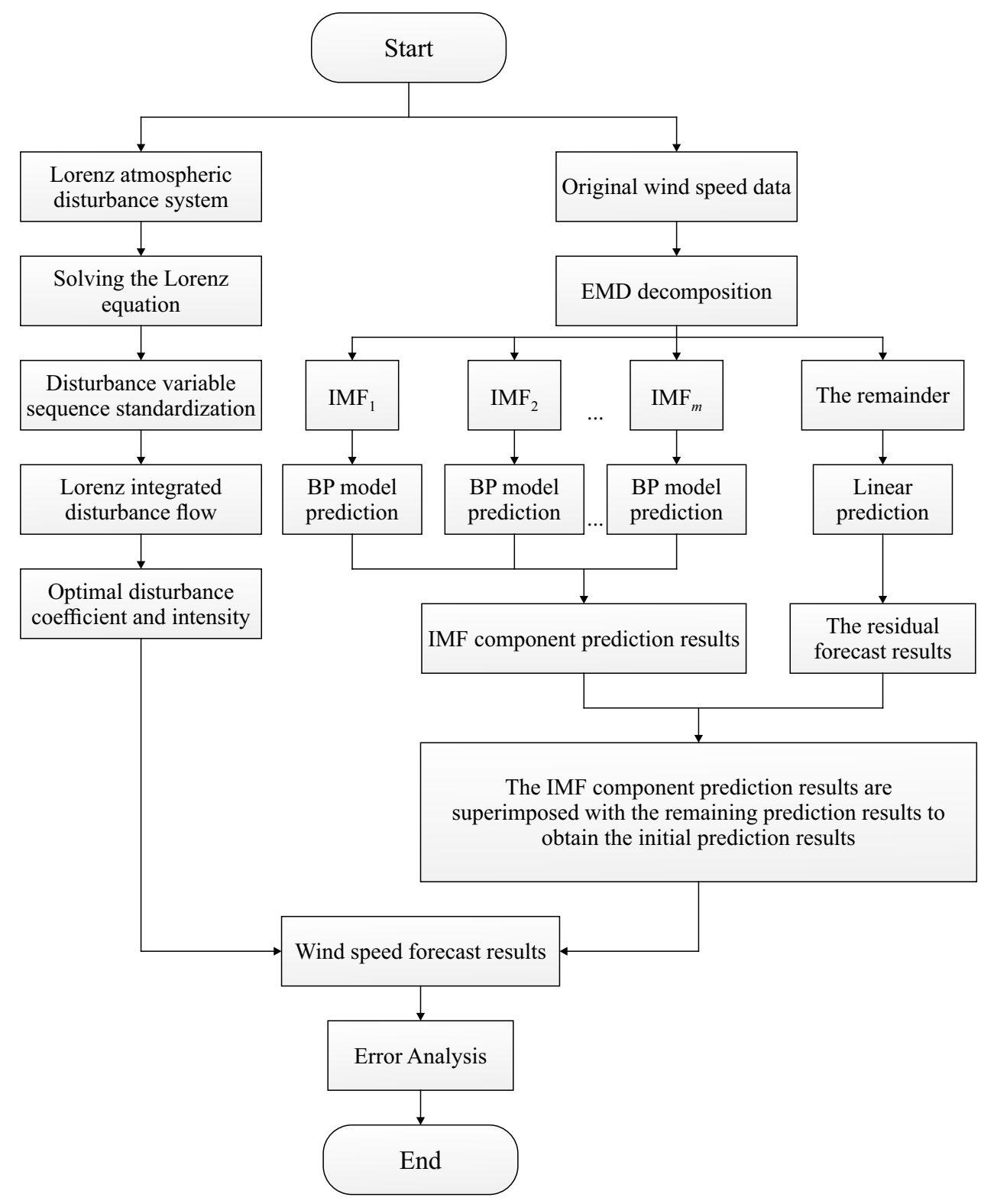

Fig. 7. The flow chart of LD-EMD-BP wind speed prediction model

initial wind speed prediction results. The disturbance correction formula is shown as

$$
V\left(v_{1}, \ldots, v_{n}\right)=v\left(v_{1}, \ldots, v_{n}\right)+L D\left(l_{1}, \ldots, l_{n}\right)
$$

In $(8), V\left(v_{1}, \ldots, v_{n}\right)$ indicates the preliminary wind speed prediction values; $v\left(v_{1}, \ldots, v_{n}\right)+L D\left(l_{1}, \ldots, l_{n}\right)$ indicates the preliminary wind speed prediction values. The parameter $L D\left(l_{1}, \ldots, l_{n}\right)$ is the applied Lorenz disturbance sequence, defined as

$$
L D\left(l_{1}, \ldots, l_{n}\right)=\beta \times L\left(l_{1}, \ldots, l_{n}\right) .
$$

In (9), the parameter $\beta$ is the disturbance coefficient. Its positive and negative values indicate the positive and negative reinforcement effects of the disturbance sequences respectively. The parameter $L\left(l_{1}, \ldots, l_{n}\right)$ is a contiguous sequence in the LCDF disturbance sequences, showing the disturbance intensity. The parameter $n$ is the prediction sample number.

Step 6: Error Analysis. Use the mean absolute error (MAE), the root mean squared error (RMSE) and the mean absolute percentage error (MAPE) comprehensibly evaluate the forecasting results.

$$
\begin{aligned}
M A E & =\frac{1}{k} \sum_{t=1}^{k}|V(t)-\hat{V}(t)| \\
R M S E & =\sqrt{\frac{1}{k} \sum_{t=1}^{k}(V(t)-\hat{V}(t))^{2}} \\
M A P E & =\frac{1}{k} \sum_{t=1}^{k}\left|\frac{V(t)-\hat{V}(t)}{V(t)}\right| \times 100
\end{aligned}
$$




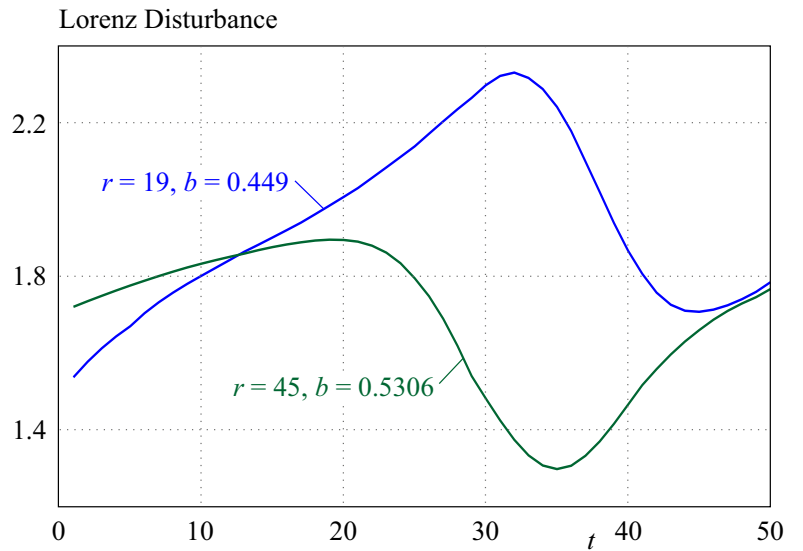

Fig. 8. The optimal disturbance coefficient and disturbance intensity of the LD-EMD-BP model

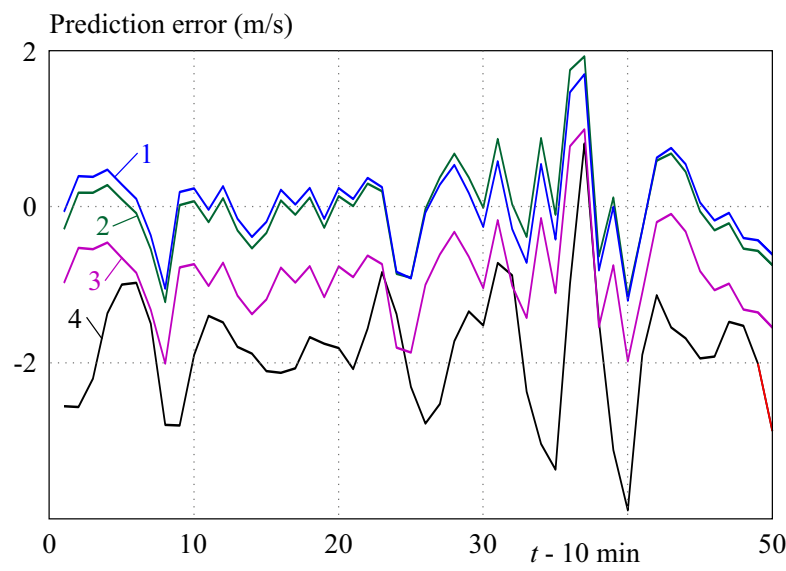

Fig. 10. The LD-EMD-BP model prediction error curve

In the three equations above, the parameter $V(t)$ is the original wind speed data and the parameter $\hat{V}(t)$ indicates the wind speed prediction value. According to the above modeling steps, the modeling flow chart of the LD-EMD-BP wind speed prediction model is shown in Fig. 7.

\section{LD-EMD-BP model wind speed prediction results analysis}

\subsection{The optimal disturbance coefficient and disturbance intensity}

The method calculating the optimal disturbance coefficient and disturbance intensity is shown in this article. To achieve the goal than the root mean square error (RMSE) between the primary prediction sequences and the real sequences is minimized, the optimal disturbance coefficient and perturbation intensity of the LD-EMDBP model are obtained through two-dimensional network search, as shown in Fig. 8. The optimal disturbance coefficients in the transient chaotic and chaotic states are

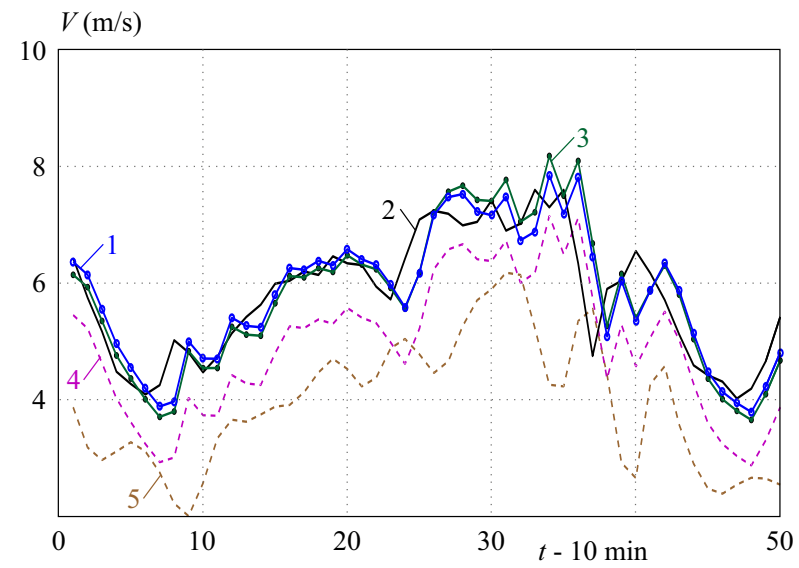

Fig. 9. The wind speed prediction curve of the LD-EMD-BP model

0.449 and 0.5306 respectively, which are positive numbers, indicating that the preliminary wind speed prediction values is smaller than original wind speed values, and a positive disturbance correction sequences needs to be applied. However, the two optimal perturbation curves are nearly symmetric. Under different motion conditions, the difference in the applied perturbation curves is large.

\section{2 wind speed forecasting results}

According to the preliminary wind speed prediction model and the obtained optimal disturbance coefficient and disturbance intensity, the wind speed prediction curve of LD-EMD-BP can be obtained, as shown in Fig. 9. The horizontal axis indicates the prediction period and the vertical axis shows the wind speed value. The black curve is the original wind speed sequences. The brown dashed line is the BP neural network wind speed forecasting results. The purple dashed line is the EMD-BP neural network wind speed forecasting result. The green solid line is the prediction result of the disturbance correction in the transient chaotic state, and the blue solid line is the prediction result of the disturbance correction in the chaotic state. The wind speed forecasting curves of the BP neural network model and EMD-BP model are all below the original wind speed curve and can describe the wind speed sequences trend. However, the wind speed forecasting curve of the EMD-BP model is closer to the original wind speed sequences, and the presented trend is basically the same as the original wind speed sequences, which fully shows that EMD decomposition can improve the BP neural network prediction performance. The wind speed prediction curve of the EMD-BP model is below the original wind speed curve, and a positive perturbation sequence is applied to make it more in line with the original wind speed movement. The green solid line and blue solid line represent the wind speed prediction results modified by Lorenz's disturbance, the Lorenz disturbance sequences under the transient chaos and the chaos have big differences, but the modified LD-EMD-BP 
model maintains the trend of the original wind speed sequences and is numerically closer to the actual wind speed sequences. This fully illustrates that the disturbance sequences obtained by the Lorenz equation under different motion states weaken the randomness of the original wind speed sequences to a certain extent, and obtain reliable prediction results, improving the wind speed forecasting accuracy.

\subsection{Error analysis for wind speed prediction models}

The prediction error of each model is obtained based on the prediction result and the actual wind speed. The error curve is shown in Fig. 10. The black curve represents the error of the BP neural network prediction model, and the purple curve represents the error of the EMD-BP model. Most of the two curves are below $y=0$. The black curve fluctuates between $-4 \mathrm{~m} / \mathrm{s}$ and $1 \mathrm{~m} / \mathrm{s}$. Purple curve fluctuates between $-2.5 \mathrm{~m} / \mathrm{s}$ and $1 \mathrm{~m} / \mathrm{s}$. The fluctuation range of the purple curve is smaller than that of the red curve. The EMD decomposition can effectively improve the BP neural network prediction performance. The green curve and the blue curve respectively represent the error of the modified wind speed prediction curve by the disturbance sequence under the transient chaos and the chaos. Both curves fluctuate around $y=0$, and the fluctuation range is small. This fully shows that the disturbance sequences under the transient chaos and chaos states have a good correction influence on the preliminary wind speed prediction results.

Table 2. The wind speed prediction model error statistics

\begin{tabular}{lcccc}
\hline Wind speed & BP & EMD-BP & \multicolumn{2}{c}{ LD-EMD-BP } \\
prediction model & & & $r=19$ & $r=45$ \\
\hline $\operatorname{MAE}(\mathrm{m} / \mathrm{s})$ & 1.8915 & 0.9404 & 0.4232 & 0.4199 \\
$\operatorname{RMSE}(\mathrm{m} / \mathrm{s})$ & 2.0172 & 1.0438 & 0.5963 & 0.5539 \\
$\operatorname{MAPE}(\%)$ & 33.19 & 16.81 & 7.51 & 7.4 \\
\hline
\end{tabular}

The mean absolute error (MAE), the root-meansquare error (RMSE) and the absolute mean percentage error (MAPE) were applied to evaluate the predicted results comprehensively. Table 2 shows the specific prediction accuracy of each model. The three error indicators of the BP neural network model are larger than those of the EMD-BP model, and the three error indicators of the EMD-BP model reduces by more than $50 \%$. At the same time, the error indicators of the BP and EMD-BP models are far greater than the prediction accuracy of the LDEMD-BP model. This not only fully indicates that after the EMD processes, the prediction BP neural network effect is significantly improved, but also illustrates the good improvement effect of the Lorenz disturbance sequences on the preliminary wind speed prediction. Compared with the MAE of BP neural network, the LD-EMD-BP models with $r=19$ and $r=45$ decreases by $77.6 \%$ and $77.8 \%$ respectively, The RMSE decreased by $70.4 \%$ and $72.5 \%$, and the MAPE dropped by at least $25.68 \%$. These data objectively show that EMD decomposition effectively improves the BP neural network prediction performance and the introduced Lorenz system significantly improves the preliminary wind speed prediction results and the model prediction accuracy. For two different atmospheric motion modalities, the Lorenz equation has different effects, but all of them modify the preliminary wind speed prediction sequences to some extent, and obtain more accurate prediction results.

\section{Conclusion and prospection}

With the continuous innovation of new energy technologies, the cost of renewable energy is rapidly declining, and the proportion of new energy sources in the global energy structure is increasing, the world energy landscape is transforming and updating. The extensive use of new energy sources will reduce the exploitation rate of fossil energy sources, ease carbon emissions, and reduce environmental pressures caused by energy consumption. In the new energy power generation systems based on wind power and solar energy, wind power is the most mature and most cost-competitive green energy power source.

The inherent random volatility of wind energy will inevitably affect the stability of wind power grid connection, thus limiting the wind power large-scale development, and improving wind speed prediction accuracy is a necessary link to ensure the wind farm safe operation. Therefore, this paper adopts wind speed data from the Chinese wind farms, introduces the Lorenz disturbance models under two kinds of movement patterns, improves the EMD-BP model, eliminates the randomness of the wind speed sequences, significantly improves the wind speed prediction accuracy, and promotes wind power large-scale grid-connected, and provides effective protection for new energy generation replace traditional energy generation. In view of the significant improvement consequent of the Lorenz disturbance system for the wind speed prediction result, in the next research work we will continue to consider the influence of the Lorenz atmospheric disturbance model on the wind power prediction based on other numerical algorithms, and further improve the prediction accuracy.

\section{Acknowledgements}

This research was supported partly by the National Natural Science Foundation of China (51637005), the Fundamental Research Funds for the Central Universities (2017MS166), the Natural Science Foundation of Hebei Province (G2016502009) and the fund of North China Electric Power University. 


\section{REFERENCES}

[1] Global Wind Energy Council (GWEC) [DB/OL]

[2] J. Z. Wang, Y. L. Song, F. Liu, and R. Hou, "Analysis Application of Forecasting Models in Wind Power Integration: A Review of Multi-Step-Ahead Wind Speed Forecasting Models", Renewable Sustainable Energy Reviews vol. 60, pp. 960-981, 2016.

[3] A. Jackson and B. Turnbull, "Identification of Particle-Laden Flow Features from Wavelet Decomposition", Physica D: Nonlinear Phenomena vol. 361, pp. 12-27, 2016.

[4] K. Y. Zhang, R. Gencay, and M. E. Yazgan, "Application of Wavelet Decomposition in Time Series Forecasting", Economics Letters vol. 158, pp. 41-46, 2016.

[5] V. Gupta and M. Mittal, "KNN PCA Classifier with Autoregressive Modelling During Different ECG Signal Interpretation", Procedia Computer Science vol. 125, pp. 18-24, 2017.

[6] E. P. Duff, A. J. Trachtenberg, C. E. Mackay, M. A. Howard, F. Wilson, S. M. Smith, and M. W. Woolrich, "Task-Driven ICA Feature Generation for Accurate Interpretable Prediction using fMRI", NeuroImage vol. 60, pp. 189-203, Mar 2016.

[7] J. Naik, P. Satapathy, and P. K. Dash, "Short-Term Wind Speed Wind Power Prediction using Hybrid Empirical Mode Decomposition Kernel Ridge Regression", Applied Soft Computing, pp. ASOC-4606, 2017.

[8] L. Y. Liu, D. R. Liu, Q. Sun, H. L. Li, and R. Wennersten, "Forecasting Power Output of Photovoltaic Systemusing a BP Network Method", Energy Procedia vol. 142, pp. 780-786, 2017.

[9] J. C. Li, D. L. Zhao, B. F. Ge, K. W. Yang, and Y. W. Chen, "A Link Prediction Method for Heterogeneous Networks Based on BP Neural Network", Physica A: Statistical Mechanics and its Application vol. 495, pp. 1-17, 2018.

[10] F. Bre, J. M. Gimenez, and V. D. Fanchinotti, "Prediction of Wind Pressure Coefficients on Building Surfaces using Artificial Neural Networks", Energy Buildings vol. 158, pp. 1429-1441, 2018.

[11] R. Rajesh, "Forecasting Supply Chain Resilience Performance using Grey Prediction", Electronic Commerce Research Applications vol. 20, pp. 42-58, 2016.

[12] A. Bezuglov and G. Comert, "Short-Term Freeway Traffic Parameter Prediction: Application of Grey System Theory Models", Expert Systems with Application vol. 62, pp. 284-292, 2016.

[13] V. Prema and K. Uma Rao, "Development of Statistical Time Series Models for Solar Power Prediction", Renewable Energy vol. 83, pp. 100-109, 2015.

[14] Y. N. Zhao, L. Ye, Z. Li, X. R. Song, Y. S. Lang, and J. Su, "A Novel Bidirectional Mechanism Based on Time Series Model for Wind Power Forecasting", Applied Energy vol, 177, pp. 793-803, 2016.

[15] A. Khosravi, R. N. N. Koury, L. Machado, and J. J. G. Pabon, "Prediction of Wind Speed Wind Direction using Artificial Neural Network", Sustainable Energy Technologies Assessments vol. 25, pp. 146-160, 2018.

[16] S. X. Zhang, Z. P. Zhou, X. M. Chen, Y. Hu, and L. D. Yang, "PDHS-SVM: A Prediction Method for Plant DNase I Hypersensitive Sites Based on Support Vector Machine", Journal of Theoretical Biology vol. 426, pp. 126-133, 2017.

[17] Y. G. Zhang, J. Y. Yang, K. C. Wang, Z. P. Wang, and Y. D. Wang, "Improved Wind Prediction Based on the Lorenz System", Renewable Energy vol. 81, pp. 219-226, 2015.
[18] Y. G. Zhang, P. H. Wang, P. L. Cheng, and S. Lei, "Wind Speed Prediction with Wavelet Time Series Based on Lorenz Disturbance", Advances in Electrical Computer Engineering vol. 17, pp. 107-114, 2017.

[19] J. Naik, P. Satapathy, and P. K. Dash, "Short-Term Wind Speed Wind Power Prediction using Hybrid Empirical Mode Decomposition Kernel Ridge Regression", Applied Soft Computing, pp. ASOC-4606, 2017.

20] C. Zhang, H. K. Wei, J. S. Zhao, T. H. Liu, T. T. Zhu, and K. J. Zhang, "Short-Term Wind Speed Forecasting using Empirical Mode Decomposition Feature Selection", Renewable Energy vol. 96, pp. 727-737, 2016.

[21] W. Y. Duan, Y. Han, L. M. Huang, B. B. Zhao, and M. H. Wang, "A Hybrid EMD-SVR Model for the Short-Term Prediction of Significant Wave Height", Ocean Engineering, vol. 124, pp. 54-73, 2016.

[22] Y. G. Zhang, Y. Xu, and Z. P. Wang, "GM $(1,1)$ Gray Prediction of Lorenz Chaotic System" Chaos Solitons and Fractals vol. 42, pp. 1003-1009, 2009.

$23]$ Y. G. Zhang, J. Y. Yang, K. C. Wang, Z. P. Wang, and Y. D. Wang, "Lorenz Wind Disturbance Model Based on Grey Generated Components", Energies vol. 7, pp. 7178-7193, 2014.

[24] Y. G. Zhang, J. Y. Yang, K. C. Wang, Z. P. Wang, and Y. D. Wang, "Wind Power Prediction Considering Nonlinear Atmospheric Disturbance", Energies vol. 8, pp. 475-478, 2014.

[25] Y. G. Zhang, C. H. Zhang, Y. Zhao, and S. Gao, "Wind Speed Prediction with RBF Neural Network Based on PCA and ICA", Journal of Electrical Engineering vol. 69, no. 2, pp. 148-155, 2018 .

Received 2 January 2019

Yagang Zhang received his BS degree in Department of Mathematics from Yunnan University, Kunming, Yunnan Province, China in 2001 and the MS degree in Center for Nonlinear Complex Systems from Yunnan University, Kunming, Yunnan Province, China in 2004. He received the $\mathrm{PhD}$ degree from North China Electric Power University, China, in 2011. At present, he is an associate professor in the State Key Laboratory of Alternate Electrical Power System with Renewable Energy Sources, North China Electric Power University, Baoding, Hebei Province, China. His current research topics include renewable energy source, relay protection of power system and nonlinear complex system theory.

Guifang Pan received her bachelor degree from Department of Mathematics and Physics from North China Electric Power University, Baoding, Hebei Province, China in 2018. At present, she is a Master in applied statistics at North China Electric Power University. Her research area is new energy forecast.

Chenhong Zhang graduated from Handan College, Handan, Hebei Province, China in 2016 and obtained a bachelor degree in mathematics and applied mathematics. At present, she is a Master at Applied Mathematics in North China Electric Power University. Her research area is new energy forecast.

Yuan Zhao graduated from the Hubei Institute of Technology, Xianning, Hubei Province, China in 2017 and obtained a bachelor degree in applied statistics. At present, she is a Master in applied statistics at North China Electric Power University. Her research area is new energy forecast. 